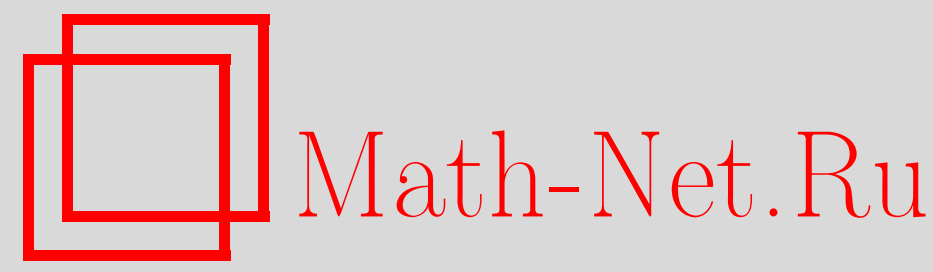

Е. Ю. Панов, О наибольших и наименьших обобщенных энтропийных решениях задачи Коши для квазилинейного уравнения первого порядка, Матем. сб., 2002, том 193, номер 5, 95-112

DOI: https://doi.org/10.4213/sm653

Использование Общероссийского математического портала Math-Net.Ru подразумевает, что вы прочитали и согласны с пользовательским соглашением

http://www. mathnet.ru/rus/agreement

Параметры загрузки:

IP: 54.237 .206 .68

26 апреля 2023 г., 14:46:25

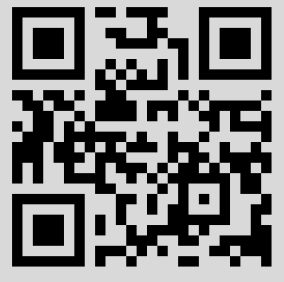




\section{Е.Ю. Панов \\ О наибольших и наименьших обобшенных энтропийных решениях задачи Коши для квазилинейного уравнения первого порядка}

Доказано существование наибольшего и наименьшего обобщенных энтропийных решений (о.э.р.) задачи Коши для квазилинейного уравнения первого порядка в общем случае, когда вектор потока лишь непрерьвен и свойство единственности о.э.р. может нарушаться. Даны некоторые полезные приложения. В частности, установлена единственность о.э.р. в случае периодических по $n-1$ пространственному вектору входных данных ( $n$ - число пространственных переменных).

Библиограбол: 18 названий.

\section{§1. Введение}

Рассмотрим задачу Коши для квазилинейного уравнения первого порядка

$$
u_{t}+\operatorname{div}_{x} \varphi(u)=g(t, x)
$$

$\varphi=\left(\varphi_{1}, \ldots, \varphi_{n}\right), u=u(t, x),(t, x) \in \Pi_{T}=(0, T) \times \mathbb{R}^{n}, T>0$, с начальным условием

$$
u(0, x)=u_{0}(x) \in L^{\infty}\left(\mathbb{R}^{n}\right) .
$$

Предполагается, что функция источника $g=g(t, x) \in L_{\mathrm{loc}}^{1}\left(\Pi_{T}\right)$ в $(1)$ удовлетворяет условию:

$$
g(t, \cdot) \in L^{\infty}\left(\mathbb{R}^{n}\right) \text { для п.в. } t \in(0, T) \text { и } \int_{0}^{T}\|g(t, \cdot)\|_{\infty} d t<\infty .
$$

Функции потока $\varphi_{i}(u)$ предполагаются лишп непрерывными:

$$
\varphi_{i}(u) \in C(\mathbb{R}), \quad i=1, \ldots, n
$$

Работа вьполнена при поддержке Российского фонда фундаментальных исследований (гранты №№ 00-01-00921 и 02-01-00483) и гранта Президента РФ (№ 00-15-99254).

$$
\text { (C) Е. Ю. ПАнов } 2002
$$


ОПРЕДЕЛЕНИЕ 1 (С.Н. Кружков, [1], [2]). Ограниченная измеримая функция $u=u(t, x)$ называется обобщенным әнтропийным решением (коротко - о.э.р.) задачи Коши (1), (2), если:

a) $\forall k \in \mathbb{R}$

$$
|u-k|_{t}+\operatorname{div}_{x}[\operatorname{sign}(u-k)(\varphi(u)-\varphi(k))] \leqslant \operatorname{sign}(u-k) g
$$

в смысле распределений на $\Pi_{T}\left(\right.$ в $\left.\mathscr{D}^{\prime}\left(\Pi_{T}\right)\right)$;

b) ess $\lim _{t \rightarrow 0+} u(t, \cdot)=u_{0}$ в $L_{\text {loc }}^{1}\left(\mathbb{R}^{n}\right)$, т.е. существует множество $\mathscr{E} \subset(0, T)$ полной меры Лебега такое, что $u(t, \cdot) \in L_{\mathrm{loc}}^{1}\left(\mathbb{R}^{n}\right), t \in \mathscr{E}$, и $u(t, \cdot) \rightarrow u_{0}$ в $L_{\mathrm{loc}}^{1}\left(\mathbb{R}^{n}\right)$ при $t \rightarrow 0+, t \in \mathscr{E}$.

Условие (4) означает, что для любой пробной функции $f=f(t, x) \in C_{0}^{\infty}\left(\Pi_{T}\right)$, $f \geqslant 0$,

$$
\int_{\Pi}\left\{|u-k| f_{t}+\operatorname{sign}(u-k)\left[\left(\varphi(u)-\varphi(k), \nabla_{x} f\right)+g f\right]\right\} d t d x \geqslant 0
$$

здесь и ниже $(\cdot, \cdot)$ означает скалярное умножение на $\mathbb{R}^{n}$.

Хорошо известно (см., например, [2], [3]), что условие (4) можно записать в эквивалентной форме: для любой выпуклой функции $\eta(u) \in C^{1}(\mathbb{R})$ (энтропии)

$$
\eta(u)_{t}+\operatorname{div}_{x} \psi(u) \leqslant \eta^{\prime}(u) g \quad \text { в } \mathscr{D}^{\prime}\left(\Pi_{T}\right)
$$

где $\psi(u)=\left(\psi_{1}(u), \ldots, \psi_{n}(u)\right)$ - соответствующий вектор потока энтропии, определяемый равенством

$$
\psi(u)=\eta^{\prime}(u) \varphi(u)-\int_{0}^{u} \varphi(\sigma) d \eta^{\prime}(\sigma)
$$

(так что в случае гладкого потока $\varphi(u) \in C^{1}\left(\mathbb{R}, \mathbb{R}^{n}\right)$ вектор $\psi(u)$ определен, с точностью до аддитивной константы, равенством $\left.\psi^{\prime}(u)=\eta^{\prime}(u) \varphi^{\prime}(u)\right)$.

Положив в (4) $k= \pm\|u\|_{\infty}$, легко вывести, что $u_{t}+\operatorname{div}_{x} \varphi(u)=g$ в $\mathscr{D}^{\prime}\left(\Pi_{T}\right)$ и $u(t, x)$ удовлетворяет уравнению (1) в смысле распределений (т.е. действительно является обобшенным решением).

Исследование задачи (1), (2) в случае лишь непрерывного потока $\varphi(u)$ было начато в работах [3]-[5]. С тех пор теория задачи (1), (2) значительно продвинута.

Напомним понятия и результаты, которые будут использоваться ниже. Хорошо известно, что о.э.р. задачи (1), (2) всегда сушествует (доказательство содержится, например, в [6]-[8]).

В рассматриваемом случае, когда вектор потока предполагается лишш непрерывным, появляется эффект бесконечной скорости распространения начального возмущения, которьй может приводить к неединственности о.э.р. задачи (1), (2), если число пространственных переменных $n>1$. Соответствующие примеры были впервые построены в [7], [9], [10] для случая $n=2, g \equiv 0$. Заметим, что для непрерывного по Липшицу потока единственность о.э.р. хорошо известна (см. [1], [2]). 
Некоторые достаточные условия единственности найдены в [7], [9], [10]. Более того, при этих достаточных условиях в [10] (см. также [11], [12]) доказан приниип сравнения для обобшенных энтропийных суб- и супер-решений.

Напомним определение обобщенных энтропийных суб- и супер-решений (см. [3], [9], [11]-[15]). Пусть $f^{+}=\max (f, 0), f^{-}=\max (-f, 0)$. Введем обозначение $\chi_{u>v}$ для характеристической функции множества независимых переменных, на котором выполнено сравнение $u>v$ для двух данных функций $u$ и $v$. Аналогично определяются функции $\chi_{u<v}, \chi_{u=v}$ и т. д. Пусть $\operatorname{sign}^{+}(r)=\chi_{r>0}-$ функция Хевисайда, $\operatorname{sign}^{-}(r)=-\operatorname{sign}^{+}(-r)$.

ОПРЕДЕЛЕниЕ 2. Ограниченная измеримая функция $u(t, x)$ на $\Pi_{T}$ называется обобщенным энтропийным суб-решением (коротко - о.э.суб-р.) задачи Коши (1), (2), если

1) $\forall k \in \mathbb{R}$

$$
\left((u-k)^{+}\right)_{t}+\operatorname{div}_{x}\left[\operatorname{sign}^{+}(u-k)(\varphi(u)-\varphi(k))\right] \leqslant \operatorname{sign}^{+}(u-k) g \text { в } \mathscr{D}^{\prime}\left(\Pi_{T}\right),
$$

2) ess $\lim _{t \rightarrow 0+}\left(u(t, x)-u_{0}(x)\right)^{+}=0$ в $L_{\mathrm{loc}}^{1}\left(\mathbb{R}^{n}\right)$.

ОПРЕДЕЛЕНИЕ 3 . Ограниченная измеримая функция $u(t, x)$ на $\Pi_{T}$ называется обобщенным энтропийным супер-решением (коротко - о.э.супер-р.) задачи Коши (1), (2), если

1) $\forall k \in \mathbb{R}$

$\left((u-k)^{-}\right)_{t}+\operatorname{div}_{x}\left[\operatorname{sign}^{-}(u-k)(\varphi(u)-\varphi(k))\right] \leqslant \operatorname{sign}^{-}(u-k) g$ в $\mathscr{D}^{\prime}\left(\Pi_{T}\right)$,

2) ess $\lim _{t \rightarrow 0+}\left(u(t, x)-u_{0}(x)\right)^{-}=0$ в $L_{\mathrm{loc}}^{1}\left(\mathbb{R}^{n}\right)$.

ЗАмечание 1 (см. [6], [10], [11], [16]). а) Условия (7) и (8) эквивалентны требованию, что (5) выполнено для всех неубываюших (соответственно невозрастающих) выпуклых функций $\eta(u) \in C^{1}(\mathbb{R})$.

b) Функция $u=u(t, x) \in L^{\infty}(\Pi)$ является о.э.супер-р. задачи (1), (2) тогда и только тогда, когда функция $-u$ является о.э.суб-р. задачи Коши для уравнения $u_{t}-\operatorname{div}_{x} \varphi(-u)=-g$ с начальньми данньми $-u_{0}(x)$.

c) Функция $u(t, x) \in L^{\infty}(\Pi)$ - о.э.р. задачи $(1),(2)$ тогда и только тогда, когда она является о.э.суб-р. и о.э.супер-р. этой задачи одновременно.

d) Пусть $y=A x$ - невырожденное линейное преобразование на $\mathbb{R}^{n}$. Тогда $u(t, x)$ - о.э.суб-р. (о.э.супер-р., о.э.p.) задачи $(1),(2)$ тогда и только тогда, когда функция $\bar{u}(t, y)=u\left(t, A^{-1} y\right)$ является о.э.суб-р. (соответственно о.э.супер-р., о.э.р.) задачи

$$
u_{t}+\operatorname{div}_{y} \bar{\varphi}(u)=\bar{g}, \quad u(0, y)=u_{0}\left(A^{-1} y\right),
$$

где $\bar{g}(t, y)=g\left(t, A^{-1} y\right)$ и вектор потока $\bar{\varphi}(u)=A^{*} \varphi(u), A^{*}: \mathbb{R}^{n} \rightarrow \mathbb{R}^{n}-$ сопряженное преобразование.

Нам понадобится также следующий результат, содержащийся в [6], [12]. 
ПРЕДЛОЖЕНИЕ 1. Пусть $u(t, x) u \widetilde{u}(t, x)$ - o.ә.суб-p. $u$ о.э.супер-p. задачu $(1),(2)$ с начальными функииями $u_{0}(x)$ и $\widetilde{u}_{0}(x)$ и функииями источника $g(t, x)$ и $\widetilde{g}(t, x)$ соответственно.

Предположим также, что выполнено одно из следующих условий:

А) одна из функций и или $\widetilde{u}$ не зависит от $x$, m.е. $u \equiv u(t)$ или $\widetilde{u} \equiv \widetilde{u}(t)$ n.в. на $\Pi_{T}$;

В) $\mu_{n+1}\left\{(t, x) \in \Pi_{T}: u(t, x)>\widetilde{u}(t, x)\right\}<\infty$, әде $\mu_{r}, r \in \mathbb{N}$, обозначает г-мерную меру Лебега.

Тогда для п.в. $t \in(0, T)$

$$
\begin{aligned}
\int_{\mathbb{R}^{n}} & (u(t, x)-\widetilde{u}(t, x))^{+} d x \\
& \leqslant \int_{\mathbb{R}^{n}}\left(u_{0}(x)-\widetilde{u}_{0}(x)\right)^{+} d x+\int_{\Pi_{t}}(g(\tau, x)-\widetilde{g}(\tau, x))^{+} \chi_{u} \geqslant \widetilde{u} d \tau d x .
\end{aligned}
$$

В частности, если $u_{0} \leqslant \widetilde{u}_{0}$ n.в. на $\mathbb{R}^{n}, g \leqslant \widetilde{g}$ п.в. на $\Pi_{T}$, то $u \leqslant \widetilde{u}$ п.в.на $\Pi_{T}$ (принцип сравнения).

Применяя принцип сравнения к о.э.р. и задачи (1), (2), о.э.суб-р.

$$
u_{-}(t)=\operatorname{essinf} u_{0}(x)+\int_{0}^{t} \underset{x \in \mathbb{R}^{n}}{\operatorname{essinf}} g(\tau, x) d \tau
$$

и о.э.супер-р.

$$
u_{+}(t)=\operatorname{ess} \sup u_{0}(x)+\int_{0}^{t} \underset{x \in \mathbb{R}^{n}}{\operatorname{ess} \sup } g(\tau, x) d \tau
$$

этой же задачи (заметим, что ввиду (3) функции $u_{-}(t)$ и $u_{+}(t)$ ограничены), легко вывести приниип максимума (см. [11], [12]):

СлЕДСТВИЕ 1. Если $u=u(t, x)$ - о.э.р. задачи $(1),(2)$, то для n.в. $(t, x) \in \Pi_{T}$ $\operatorname{ess} \inf u_{0}(x)+\int_{0}^{t} \underset{x \in \mathbb{R}^{n}}{\operatorname{essinf}} g(\tau, x) d \tau \leqslant u(t, x) \leqslant \operatorname{ess} \sup u_{0}(x)+\int_{0}^{t} \underset{x \in \mathbb{R}^{n}}{\operatorname{ess} \sup } g(\tau, x) d \tau$

Для двух о.э.р. $u$ и $\widetilde{u}$ задачи (1), (2), удовлетворяющих требованию А) предложения 1, можно поменять местами эти функции в неравенстве (9) и получить

СЛЕДСТВИЕ 2 (см. [11], [12]). Пусть $u=u(t, x), \widetilde{u}=\widetilde{u}(t)-$ о.э.p. задачи (1), (2) с начальными данными $u_{0}(x)$ и $\widetilde{u}_{0}(x) \equiv \widetilde{u}(0)$ и функииями источника $g(t, x)$ и $\widetilde{g}(t, x) \equiv \widetilde{g}(t)$ соответственно. Тогда для п.в. $t \in(0, T)$

$$
\int_{\mathbb{R}^{n}}|u(t, x)-\widetilde{u}(t)| d x \leqslant \int_{\mathbb{R}^{n}}\left|u_{0}(x)-\widetilde{u}(0)\right| d x+\int_{\Pi_{t}}|g(\tau, x)-\widetilde{g}(\tau)| d \tau d x .
$$

В [6], [11], [12] были исследованы некоторые специальные случаи единственности в классе суммируемых по пространственньм переменным решений. Установленное в этих работах существование наибольшего и наименьшего о.э.р. играет важную роль для изучения проблемы. Существование наибольшего и наименьшего 
о.э.р. было доказано в предположении, что начальные данные и функция источника в определенном смысле "исчезают" при $x \rightarrow \infty$.

В $\S 2$ мы докажем существование наибольшего и наименњшего решений в общем случае и дадим некоторые полезные приложения. В частности, мы покажем, что наибольшее (наименьшее) о.э.р. совпадает с наибольшим о.э.суб-р. (соответственно с наименьшим о.э.супер-р.), существование которых установлено в [16]. В 33 доказывается единственность о.э.р. задачи $(1),(2)$ в случае, когда входные данные задачи являются периодическими по $n-1$ пространственньм переменным функциями. Таким образом, дается обобщение соответствующего результата из [16].

Следует отметить, что результаты, аналогичные изложенным выше, верны и для стационарной задачи

$$
u+\operatorname{div}_{x} \varphi(u)=g(x), \quad u=u(x), \quad x \in \mathbb{R}^{n}
$$

изучение которой проводилось в статьях [6], [11], [12] параллельно с исследованием задачи Коши.

В настоящей работе мы ограничимся лиш случаем задачи Коши $(1),(2)$, подчеркнув, что доказательства основных результатов сохраняются (с естественными изменениями) и для соответствующей стационарной задачи.

\section{§2. Основные теоремы}

Дадим следующую полезную переформулировку определения 1.

Пусть $\bar{\Pi}_{T}=[0, T) \times \mathbb{R}^{n}, C_{0}^{\infty}\left(\bar{\Pi}_{T}\right)-$ подпространство функций из $C^{\infty}\left(\bar{\Pi}_{T}\right)$ с компактным носителем (который может иметь непустое пересечение с начальной гиперплоскостью $\{t=0\})$.

ПРЕДЛОЖЕНИЕ 2. Функиия $u=u(t, x) \in L^{\infty}\left(\Pi_{T}\right)$ является о.э.р. задачи (1), (2) тогда и только тогда, когда для любого $k \in \mathbb{R}$ и любой пробной функиии $f=f(t, x) \in C_{0}^{\infty}\left(\bar{\Pi}_{T}\right), f \geqslant 0$,

$$
\begin{gathered}
\int_{\Pi_{T}}\left\{|u-k| f_{t}+\operatorname{sign}(u-k)\left[\left(\varphi(u)-\varphi(k), \nabla_{x} f\right)+g f\right]\right\} d t d x \\
\quad+\int_{\mathbb{R}^{n}}\left|u_{0}(x)-k\right| f(0, x) d x \geqslant 0 .
\end{gathered}
$$

ДокАЗАТЕЛьство. Пусть $u=u(t, x)$ - о.э.р. задачи $(1),(2) ; k \in \mathbb{R}$, $f=f(t, x) \in C_{0}^{\infty}\left(\bar{\Pi}_{T}\right), f \geqslant 0$. Выберем функцию $\beta(s) \in C_{0}^{\infty}(\mathbb{R}), \operatorname{supp} \beta(s) \subset[0,1]$, $\beta(s) \geqslant 0, \quad \int \beta(s) d s=1$, и положким при $\nu \in \mathbb{N} \quad \delta_{\nu}(s)=\nu \beta(\nu s), \theta_{\nu}(t)=$ $\int_{-\infty}^{t} \delta_{\nu}(s) d s$. Понятно, что последовательность $\delta_{\nu}(s)$ сходится к $\delta$-мере Дирака в $\mathscr{D}^{\prime}(\mathbb{R})$ при $\nu \rightarrow \infty ; 0 \leqslant \theta_{\nu}(t) \leqslant 1, \theta_{\nu}(t)=0$ при $t \leqslant 0, \theta_{\nu}(t)=1$ при $t \geqslant 1 / \nu$ и, следовательно, последовательность $\theta_{\nu}(t)$ сходится поточечно при $\nu \rightarrow \infty \mathrm{k}$ функции Хевисайда $\operatorname{sign}^{+}(t)$. 
Применяя энтропийное неравенство (4) к пробной функции

$$
f_{\nu}=f_{\nu}(t, x)=f(t, x) \theta_{\nu}\left(t-t_{0}\right) \in C_{0}^{\infty}\left(\Pi_{T}\right), \quad t_{0} \in(0, T),
$$

мы получаем, что

$$
\begin{aligned}
& \int_{0}^{T} \delta_{\nu}\left(t-t_{0}\right) \int_{\mathbb{R}^{n}}|u(t, x)-k| f(t, x) d x d t \\
& \quad+\int_{\Pi_{T}}\left\{|u-k| f_{t}+\operatorname{sign}(u-k)\left[\left(\varphi(u)-\varphi(k), \nabla_{x} f\right)+g f\right]\right\} \theta_{\nu}\left(t-t_{0}\right) d t d x \geqslant 0 .
\end{aligned}
$$

Пусть $\mathscr{E}_{1}-$ множество общих точек Лебега для функций

$$
t \mapsto F(k, t)=\int_{\mathbb{R}^{n}}|u(t, x)-k| f(t, x) d x,
$$

где $k \in \mathbb{Q}$. Ввиду счетности $\mathbb{Q}$ множество $\mathscr{E} 1$ имеет полную меру Лебега на $(0, T)$. Очевидно, отображение $k \mapsto F(k, t) \in L^{\infty}([0, T])$ непрерывно, равномерно по $t \in[0, T]$, и так как $\mathbb{Q}$ всюду плотно, мы заключаем, что точки $t \in \mathscr{E}_{1}$ - точки Лебега функций $F(k, t)$ для всех $k \in \mathbb{R}$. Пусть $\mathscr{E}_{2} \subset(0, T)$-множество полной меры из определения 1 такое, что $u(t, \cdot) \in L_{\text {loc }}^{1}\left(\mathbb{R}^{n}\right), t \in \mathscr{E} 2$, и $u(t, \cdot) \rightarrow u_{0}$ в $L_{\text {loc }}^{1}\left(\mathbb{R}^{n}\right)$ при $t \rightarrow 0+, t \in \mathscr{E}_{2}$. Положим $\mathscr{E}=\mathscr{E}_{1} \cap \mathscr{E}_{2}$. Ясно, что $\mathscr{E}$ является множеством полной меры на $(0, T)$, и если $t_{0} \in \mathscr{E}$, то в пределе при $\nu \rightarrow \infty$ соотношение $(11)$ преобразуется к неравенству

$$
\begin{aligned}
& \int_{\mathbb{R}^{n}}\left|u\left(t_{0}, x\right)-k\right| f\left(t_{0}, x\right) d x d t \\
& \quad+\int_{\Pi_{T}}\left\{|u-k| f_{t}+\operatorname{sign}(u-k)\left[\left(\varphi(u)-\varphi(k), \nabla_{x} f\right)+g f\right]\right\} \operatorname{sign}^{+}\left(t-t_{0}\right) d t d x \geqslant 0,
\end{aligned}
$$

откуда после перехода к пределу при $t_{0} \rightarrow 0, t_{0} \in \mathscr{E}$ (с учетом требования b) определения 1), вытекает (10).

Для доказательства обратной импликации предположим, что функция $u=$ $u(t, x) \in L^{\infty}\left(\Pi_{T}\right)$ удовлетворяет условию (10). Взяв в этом условии пробные функции из $C_{0}^{\infty}\left(\Pi_{T}\right)$, мы немедленно получим, что

$$
|u-k|_{t}+\operatorname{div}_{x}[\operatorname{sign}(u-k)(\varphi(u)-\varphi(k))] \leqslant \operatorname{sign}(u-k) g \quad \text { в } \quad \mathscr{D}^{\prime}\left(\Pi_{T}\right)
$$

и $и$ удовлетворяет требованию (4). Остается проверить лишш выполнение начального условия. Выберем функцию $\rho(x) \in C_{0}^{\infty}\left(\mathbb{R}^{n}\right), \rho(x) \geqslant 0$, и положим в (10) $f=f_{\nu}(t, x)=\rho(x)\left(1-\theta_{\nu}\left(t-t_{0}\right)\right)$, где $\nu \in \mathbb{N}, t_{0} \in(0, T), 1 / \nu<T-t_{0}$. Мы получим, что для всех $k \in \mathbb{R}$

$$
\begin{aligned}
& \int_{\mathbb{R}^{n}}\left|u_{0}(x)-k\right| \rho(x) d x-\int_{0}^{T} \delta_{\nu}\left(t-t_{0}\right) \int_{\mathbb{R}^{n}}|u(t, x)-k| \rho(x) d x d t \\
& \quad+\int_{\Pi_{T}} \operatorname{sign}(u-k)\left[\left(\varphi(u)-\varphi(k), \nabla_{x} \rho\right)+g \rho\right]\left(1-\theta_{\nu}\left(t-t_{0}\right)\right) d t d x \geqslant 0 .
\end{aligned}
$$


Пусть $\mathscr{E}$ - множество общих точек Лебега для функций

$$
t \mapsto \int_{\mathbb{R}^{n}}|u(t, x)-k| \rho(x) d x, \quad k \in \mathbb{R} .
$$

Так же как и для множества $\mathscr{E} 1$, легко доказать, что $\mathscr{E}$ имеет полную меру Лебега на $(0, T)$, и если $t_{0} \in \mathscr{E}$, то в пределе при $\nu \rightarrow \infty$ из (12) следует соотношение

$$
\begin{aligned}
& \int_{\mathbb{R}^{n}}\left|u_{0}(x)-k\right| \rho(x) d x-\int_{\mathbb{R}^{n}}\left|u\left(t_{0}, x\right)-k\right| \rho(x) d x \\
& \quad+\int_{\Pi_{t_{0}}} \operatorname{sign}(u-k)\left[\left(\varphi(u)-\varphi(k), \nabla_{x} \rho\right)+g \rho\right] d t d x \geqslant 0,
\end{aligned}
$$

которое дает оценку

$$
\int_{\mathbb{R}^{n}}\left|u\left(t_{0}, x\right)-k\right| \rho(x) d x \leqslant \int_{\mathbb{R}^{n}}\left|u_{0}(x)-k\right| \rho(x) d x+C\left(t_{0}\right),
$$

где с учетом условия $(3)$

$$
C\left(t_{0}\right)=\int_{\Pi_{t_{0}}}\left[|\varphi(u)-\varphi(k)| \cdot\left|\nabla_{x} \rho\right|+\|g(t, \cdot)\|_{\infty}|\rho|\right] d t d x \underset{t_{0} \in \mathscr{E}, t_{0} \rightarrow 0+}{\longrightarrow} 0
$$

(мы используем обозначение $|v|$ для евклидовой нормы вектора $v$ ). Поэтому $\forall k \in \mathbb{R}$ и $\forall \rho(x) \in C_{0}^{\infty}\left(\mathbb{R}^{n}\right), \rho(x) \geqslant 0$,

$$
\limsup _{t_{0} \in \mathscr{E}, t_{0} \rightarrow 0+} \int_{\mathbb{R}^{n}}\left|u\left(t_{0}, x\right)-k\right| \rho(x) d x \leqslant \int_{\mathbb{R}^{n}}\left|u_{0}(x)-k\right| \rho(x) d x .
$$

Ввиду непрерывной зависимости интегралов $\int_{\mathbb{R}^{n}}\left|u\left(t_{0}, x\right)-k\right| \rho(x) d x$ от функции $\rho(x) \in L^{1}\left(\mathbb{R}^{n}\right)$ и плотности $C_{0}^{\infty}\left(\mathbb{R}^{n}\right)$ в $L^{1}\left(\mathbb{R}^{n}\right)$ предельное соотношение (13) выполнено для всех неотрицательных функций $\rho(x) \in L^{1}\left(\mathbb{R}^{n}\right)$.

Выберем при положительном $\varepsilon$ ступенчатую функцию $u_{\varepsilon}=\sum_{r=1}^{N} \alpha_{r} \chi_{A_{r}}(x)$ такую, что $\left\|u_{0}-u_{\varepsilon}\right\|_{\infty}<\varepsilon$. Здесь $\chi_{A_{r}}(x)-$ характеристические функции измеримых множеств $A_{r} \subset \mathbb{R}^{n}$. Тогда

$$
\begin{aligned}
\int_{\mathbb{R}^{n}}\left|u\left(t_{0}, x\right)-u_{0}(x)\right| \rho(x) d x & \leqslant \varepsilon\|\rho\|_{1}+\int_{\mathbb{R}^{n}}\left|u\left(t_{0}, x\right)-u_{\varepsilon}(x)\right| \rho(x) d x \\
& =\varepsilon\|\rho\|_{1}+\sum_{r=1}^{N} \int_{\mathbb{R}^{n}}\left|u\left(t_{0}, x\right)-\alpha_{r}\right| \rho \chi_{A_{r}}(x) d x
\end{aligned}
$$

и по предельному соотношению (13)

$$
\begin{aligned}
& \limsup _{t_{0} \in \mathscr{E}, t_{0} \rightarrow 0+} \int_{\mathbb{R}^{n}}\left|u\left(t_{0}, x\right)-u_{0}(x)\right| \rho(x) d x \\
& \quad \leqslant \varepsilon\|\rho\|_{1}+\sum_{r=1}^{N} \int_{\mathbb{R}^{n}}\left|u_{0}(x)-\alpha_{r}\right| \rho \chi_{A_{r}}(x) d x \\
& \quad=\varepsilon\|\rho\|_{1}+\int_{\mathbb{R}^{n}}\left|u_{0}(x)-u_{\varepsilon}(x)\right| \rho(x) d x \leqslant 2 \varepsilon\|\rho\|_{1}
\end{aligned}
$$


для любого $\varepsilon>0$. Поэтому $\forall \rho(x) \in L^{1}\left(\mathbb{R}^{n}\right), \rho(x) \geqslant 0$,

$$
\limsup _{t_{0} \in \mathscr{E}, t_{0} \rightarrow 0+} \int_{\mathbb{R}^{n}}\left|u\left(t_{0}, x\right)-u_{0}(x)\right| \rho(x) d x=0
$$

и $\operatorname{ess} \lim _{t \rightarrow 0+} u(t, \cdot)=u_{0}$ в $L_{\text {loc }}^{1}\left(\mathbb{R}^{n}\right)$. Итак, $u$ является о.э.р. задачи $(1),(2)$, что и требовалось доказать.

СлЕДСТВИЕ 3. Если функиия $u=u(t, x) \in L^{\infty}\left(\Pi_{T}\right)$ является о. ә.р. задачи (1), (2), то для любой пробной функции $f=f(t, x) \in C_{0}^{\infty}\left(\bar{\Pi}_{T}\right)$

$$
\int_{\Pi_{T}}\left[u f_{t}+\left(\varphi(u), \nabla_{x} f\right)+g f\right] d t d x+\int_{\mathbb{R}^{n}} u_{0}(x) f(0, x) d x=0 .
$$

ДоказАтельство. Рассмотрим соотношение (10) с $k= \pm M, M \geqslant\|u\|_{\infty}$. Учитывая, что, очевидно,

$$
\int_{\Pi_{T}}\left[k f_{t}+\left(\varphi(k), \nabla_{x} f\right)\right] d t d x+\int_{\mathbb{R}^{n}} k f(0, x) d x=0,
$$

мы получаем соотношение: для всех $f=f(t, x) \in C_{0}^{\infty}\left(\bar{\Pi}_{T}\right), f \geqslant 0$,

$$
\pm\left(\int_{\Pi_{T}}\left[u f_{t}+\left(\varphi(u), \nabla_{x} f\right)+g f\right] d t d x+\int_{\mathbb{R}^{n}} u_{0}(x) f(0, x) d x\right) \geqslant 0
$$

т.е.

$$
\int_{\Pi_{T}}\left[u f_{t}+\left(\varphi(u), \nabla_{x} f\right)+g f\right] d t d x+\int_{\mathbb{R}^{n}} u_{0}(x) f(0, x) d x=0
$$

для любой неотрицательной пробной функции $f$. Для завершения доказательства остается только заметить, что произвольная пробная функция $f \in C_{0}^{\infty}\left(\bar{\Pi}_{T}\right)$ является разностью двух неотрицательных функций из $C_{0}^{\infty}\left(\bar{\Pi}_{T}\right)$. Действительно, $f=(f+C) \theta-C \theta$, где константа $C>\|f\|_{\infty}$ и неотрицательная функция $\theta \in C_{0}^{\infty}\left(\bar{\Pi}_{T}\right)$ равна единице на носителе функции $f$.

ЗАмечАниЕ 2. Так же как в предложении 2, можно доказать следующие утверждения:

1) функция $u=u(t, x) \in L^{\infty}\left(\Pi_{T}\right)$ является о.э.суб-р. задачи $(1),(2)$ тогда и только тогда, когда для любого $k \in \mathbb{R}$ и любой пробной функции $f=f(t, x) \in$ $C_{0}^{\infty}\left(\bar{\Pi}_{T}\right), f \geqslant 0$,

$$
\begin{aligned}
& \int_{\Pi_{T}}\left\{(u-k)^{+} f_{t}+\operatorname{sign}^{+}(u-k)\left[\left(\varphi(u)-\varphi(k), \nabla_{x} f\right)+g f\right]\right\} d t d x \\
& \quad+\int_{\mathbb{R}^{n}}\left(u_{0}(x)-k\right)^{+} f(0, x) d x \geqslant 0
\end{aligned}
$$

2) функция $u=u(t, x) \in L^{\infty}\left(\Pi_{T}\right)$ является о.э.супер-р. задачи $(1),(2)$ тогда и только тогда, когда для любого $k \in \mathbb{R}$ и любой пробной функции $f=f(t, x) \in$ $C_{0}^{\infty}\left(\bar{\Pi}_{T}\right), f \geqslant 0$,

$$
\begin{aligned}
& \int_{\Pi_{T}}\left\{(u-k)^{-} f_{t}+\operatorname{sign}^{-}(u-k)\left[\left(\varphi(u)-\varphi(k), \nabla_{x} f\right)+g f\right]\right\} d t d x \\
& \quad+\int_{\mathbb{R}^{n}}\left(u_{0}(x)-k\right)^{-} f(0, x) d x \geqslant 0 .
\end{aligned}
$$


Tеорема 1. Существует наибольшее о.э.р. $u=u(t, x)=J^{+}\left(u_{0}, g\right)$ задачи (1), (2). Кроме того,

1) $u(t, x) \geqslant v(t, x)$ n.в. на $\Pi_{T}$ для любого о.э.суб-р. $v(t, x)$ задачи (1), (2);

2) если $u=J^{+}\left(u_{0}, g\right), v=J^{+}\left(v_{0}, h\right)$, то для п.в. $t \in(0, T)$

$$
\begin{aligned}
\int_{\mathbb{R}^{n}}(u(t, x)-v(t, x))^{+} d x \leqslant & \int_{\mathbb{R}^{n}}\left(u_{0}(x)-v_{0}(x)\right)^{+} d x \\
& +\int_{\Pi_{t}}(g(\tau, x)-h(\tau, x))^{+} d \tau d x .
\end{aligned}
$$

ДокаЗАТЕЛЬСтво. Пусть $M=\left\|u_{0}\right\|_{\infty}, M_{1}(t)=\|g(t, \cdot)\|_{\infty}$. Выберем последовательность констант $d_{\nu}, \nu \in \mathbb{N}$, такую, что $M<d_{\nu+1}<d_{\nu} \forall \nu \in \mathbb{N}$; положим для $\nu \in \mathbb{N}$

$$
u_{0, \nu}(x)=\left\{\begin{array}{ll}
u_{0}(x), & |x| \leqslant \nu, \\
d_{\nu}, & |x|>\nu,
\end{array} \quad g_{\nu}(t, x)= \begin{cases}g(t, x), & |x| \leqslant \nu \\
M_{1}(t), & |x|>\nu\end{cases}\right.
$$

и пусть $u_{\nu}=u_{\nu}(t, x)-$ о.э.р. задачи $(1),(2)$ с начальньми данными $u_{0, \nu}(x)$ и функцией источника $g_{\nu}(t, x)$ (по теореме сушествования такое о.э.р. действительно найдется). Очевидно, функция $\widetilde{u}_{\nu}(t)=d_{\nu}+\int_{0}^{t} M_{1}(s) d s$ является о.э.р. задачи $(1),(2)$ с постоянной начальной функцией $u_{0} \equiv d_{\nu}$ и функцией источника $g \equiv$ $M_{1}(t)$; кроме того, это о.э.р. не зависит от $x$. По следствию 2 для п.в. $t \in(0, T)$

$$
\begin{aligned}
\int_{\mathbb{R}^{n}}\left|u_{\nu}(t, x)-\widetilde{u}_{\nu}(t)\right| d x & \leqslant \int_{\mathbb{R}^{n}}\left|u_{0, \nu}(x)-d_{\nu}\right| d x+\int_{\Pi_{T}}\left|g_{\nu}(t, x)-M_{1}(t)\right| d t d x \\
& \leqslant C_{\nu}=\left[\left(M+d_{\nu}\right)+2 \int_{0}^{T} M_{1}(t) d t\right] \mu_{n}\left(B_{\nu}\right)<+\infty
\end{aligned}
$$

здесь $B_{\nu}$ - шар радиуса $\nu$ в $\mathbb{R}^{n}$. Заметим, что $\widetilde{u}_{\nu+1}(t)-\widetilde{u}_{\nu}(t) \equiv d_{\nu+1}-d_{\nu}$. Используя (16), мы выводим тогда, что для любого положительного $h<d_{\nu}-d_{\nu+1}$ для п.в. $t \in(0, T)$

$$
\begin{aligned}
\int_{\mathbb{R}^{n}} & \left(u_{\nu+1}(t, x)-u_{\nu}(t, x)+h\right)^{+} d x \\
& =\int_{\mathbb{R}^{n}}\left(u_{\nu+1}(t, x)-\widetilde{u}_{\nu+1}(t)+\widetilde{u}_{\nu}(t)-u_{\nu}(t, x)+h+d_{\nu+1}-d_{\nu}\right)^{+} d x \\
& \leqslant \int_{\mathbb{R}^{n}}\left(u_{\nu+1}(t, x)-\widetilde{u}_{\nu+1}(t)+\widetilde{u}_{\nu}(t)-u_{\nu}(t, x)\right)^{+} d x \\
& \leqslant \int_{\mathbb{R}^{n}}\left|u_{\nu+1}(t, x)-\widetilde{u}_{\nu+1}(t)\right| d x+\int_{\mathbb{R}^{n}}\left|u_{\nu}(t, x)-\widetilde{u}_{\nu}(t)\right| d x \\
& \leqslant C_{\nu}+C_{\nu+1}<+\infty .
\end{aligned}
$$


По этой оценке

$$
\begin{aligned}
\mu_{n+1} & \left\{(t, x) \in \Pi_{T}: u_{\nu+1}(t, x)>u_{\nu}(t, x)\right\} \\
& =\mu_{n+1}\left\{(t, x) \in \Pi_{T}:\left(u_{\nu+1}(t, x)-u_{\nu}(t, x)+h\right)^{+}>h\right\} \\
& \leqslant \frac{1}{h} \int_{0}^{T} \int_{\mathbb{R}^{n}}\left(u_{\nu+1}(t, x)-u_{\nu}(t, x)+h\right)^{+} d x d t \leqslant\left(C_{\nu}+C_{\nu+1}\right) \frac{T}{h}<\infty
\end{aligned}
$$

и из предложения $1 \mathrm{~B})$ и того факта, что $u_{0, \nu+1}(x) \leqslant u_{0, \nu}(x)$ п.в. на $\mathbb{R}^{n}, g_{\nu+1}(t, x) \leqslant$ $g_{\nu}(t, x)$ п.в. на $\Pi_{T}$, следует оценка: для п.в. $t \in(0, T)$

$$
\begin{aligned}
\int_{\mathbb{R}^{n}}\left(u_{\nu+1}(t, x)-u_{\nu}(t, x)\right)^{+} d x \leqslant & \int_{\mathbb{R}^{n}}\left(u_{0, \nu+1}(x)-u_{0, \nu}(x)\right)^{+} d x \\
& +\int_{\Pi_{T}}\left(g_{\nu+1}(t, x)-g_{\nu}(t, x)\right)^{+} d t d x=0 .
\end{aligned}
$$

Таким образом, $u_{\nu+1}(t, x) \leqslant u_{\nu}(t, x)$ п.в. на $\Pi_{T}$ для всех $\nu \in \mathbb{N}$. По следствию 1 последовательность $u_{\nu}(t, x)$ также существенно ограничена снизу:

$$
u_{\nu}(t, x) \geqslant-M-\int_{0}^{T} M_{1}(t) d t \text { п.в. на } \Pi_{T}
$$

Поэтому для п.в. $(t, x) \in \Pi_{T}$ определена предельная функция $\lim _{\nu \rightarrow \infty} u_{\nu}(t, x)=$ $u(t, x) \in L^{\infty}\left(\Pi_{T}\right)$. Ясно, что $u_{\nu}(t, x) \rightarrow u(t, x)$ в $L_{\mathrm{loc}}^{1}\left(\Pi_{T}\right)$ при $\nu \rightarrow \infty$. По определению последовательности $u_{0, \nu}$ и $g_{\nu}$ сходятся поточечно при $\nu \rightarrow \infty$ к функциям $u_{0}$ и $g$ и в пространствах $L_{\text {loc }}^{1}\left(\mathbb{R}^{n}\right)$ и $L_{\text {loc }}^{1}\left(\Pi_{T}\right)$ соответственно.

Так как $u_{\nu}(t, x)$ является о.э.р. задачи $(1),(2)$ с $u_{0}=u_{0, \nu}, g=g_{\nu}$, то выполнено условие (10): для всех $k \in \mathbb{R}$ и всех пробных функций $f=f(t, x) \in C_{0}^{\infty}\left(\bar{\Pi}_{T}\right), f \geqslant 0$,

$$
\begin{aligned}
& \int_{\Pi_{T}}\left\{\left|u_{\nu}-k\right| f_{t}+\operatorname{sign}\left(u_{\nu}-k\right)\left[\left(\varphi\left(u_{\nu}\right)-\varphi(k), \nabla_{x} f\right)+g_{\nu} f\right]\right\} d t d x \\
& \quad+\int_{\mathbb{R}^{n}}\left|u_{0, \nu}(x)-k\right| f(0, x) d x \geqslant 0 .
\end{aligned}
$$

Переходя к пределу при $\nu \rightarrow \infty$, с учетом полученных выше предельных соотношений мы получаем, что для всех $k \in \mathbb{R}$ и всех $f=f(t, x) \in C_{0}^{\infty}\left(\bar{\Pi}_{T}\right), f \geqslant 0$,

$$
\begin{aligned}
& \int_{\Pi_{T}}\left\{|u-k| f_{t}+\operatorname{sign}(u-k)\left[\left(\varphi(u)-\varphi(k), \nabla_{x} f\right)+g f\right]\right\} d t d x \\
& \quad+\int_{\mathbb{R}^{n}}\left|u_{0}(x)-k\right| f(0, x) d x \geqslant 0,
\end{aligned}
$$

и по предложению $2 u=u(t, x)-$ о.э.р. исходной задачи $(1),(2)$.

Покажем, что $u(t, x)$ удовлетворяет требованию 1$)$ теоремы. Пусть $v=v(t, x)-$ о.э.суб-р. задачи (1), (2). Так как функция $\widetilde{v}=\widetilde{v}(t)=M+\int_{0}^{t} M_{1}(s) d s$ является 
о.э.p. (и поэтому о.э.супер-р.) этой задачи с данными $u_{0} \equiv M, g \equiv M_{1}(t)$, то по предложению 1 А) для п.в. $t \in(0, T)$

$$
\int_{\mathbb{R}^{n}}(v(t, x)-\widetilde{v}(t))^{+} d x \leqslant \int_{\mathbb{R}^{n}}\left(u_{0}(x)-M\right)^{+} d x+\int_{\Pi_{T}}\left(g(t, x)-M_{1}(t)\right)^{+} d t d x=0
$$

и $v(t, x) \leqslant \widetilde{v}(t)$ п.в. на $\Pi_{T}$. Используя это неравенство, очевидное тождество $\widetilde{v}(t)-\widetilde{u}_{\nu}(t)=M-d_{\nu}$ и оценку (16), мы устанавливаем, что для любых $\nu \in \mathbb{N}$, $h \in \mathbb{R}, 0<h<d_{\nu}-M$,

$$
\begin{aligned}
\int_{\mathbb{R}^{n}}\left(v(t, x)-u_{\nu}(t, x)+h\right)^{+} d x & \leqslant \int_{\mathbb{R}^{n}}\left(\widetilde{v}(t)-u_{\nu}(t, x)+h\right)^{+} d x \\
& \leqslant \int_{\mathbb{R}^{n}}\left(\widetilde{u}_{\nu}(t)-u_{\nu}(t, x)+h+M-d_{\nu}\right)^{+} d x \\
& \leqslant \int_{\mathbb{R}^{n}}\left(\widetilde{u}_{\nu}(t)-u_{\nu}(t, x)\right)^{+} d x \leqslant C_{\nu} .
\end{aligned}
$$

Отсюда следует, что

$$
\begin{aligned}
\mu_{n+1} & \left\{(t, x) \in \Pi_{T}: v(t, x)>u_{\nu}(t, x)\right\} \\
& =\mu_{n+1}\left\{(t, x) \in \Pi_{T}:\left(v(t, x)-u_{\nu}(t, x)+h\right)^{+}>h\right\} \\
& \leqslant \frac{1}{h} \int_{0}^{T} \int_{\mathbb{R}^{n}}\left(v(t, x)-u_{\nu}(t, x)+h\right)^{+} d x d t \leqslant C_{\nu} \frac{T}{h}<\infty .
\end{aligned}
$$

Снова применяя предложение 1 В), мы выводим, что для п.в. $t \in(0, T)$

$$
\begin{aligned}
\int_{\mathbb{R}^{n}}\left(v(t, x)-u_{\nu}(t, x)\right)^{+} d x \leqslant & \int_{\mathbb{R}^{n}}\left(u_{0}(x)-u_{0, \nu}(x)\right)^{+} d x \\
& +\int_{\Pi_{T}}\left(g(t, x)-g_{\nu}(t, x)\right)^{+} d t d x=0 .
\end{aligned}
$$

Следовательно, $v(t, x) \leqslant u_{\nu}(t, x)$ п.в. на $\Pi_{T}$ для всех $\nu \in \mathbb{N}$ и, переходя к пределу при $\nu \rightarrow \infty$, получаем, что $v(t, x) \leqslant u(t, x)$ п.в. на П $T$. Итак, условие 1$)$ теоремы вьполнено и, в частности, $u(t, x)=J^{+}\left(u_{0}, g\right)$ является наибольшим о.э.р. нашей задачи. Осталось доказать свойство 2).

Пусть $M=\max \left(\left\|u_{0}\right\|_{\infty},\left\|v_{0}\right\|_{\infty}\right), M_{1}(t)=\max \left(\|g(t, \cdot)\|_{\infty},\|h(t, \cdot)\|_{\infty}\right)$. Выберем последовательность $d_{\nu}, \nu \in \mathbb{N}$, так, что $M<d_{\nu+1}<d_{\nu} \forall \nu \in \mathbb{N}$, и положим при $\nu \in \mathbb{N}$

$$
\begin{aligned}
& u_{0, \nu}(x)=\left\{\begin{array}{ll}
u_{0}(x), & |x| \leqslant \nu, \\
d_{\nu}, & |x|>\nu,
\end{array} \quad g_{\nu}(t, x)= \begin{cases}g(t, x), & |x| \leqslant \nu, \\
M_{1}(t), & |x|>\nu,\end{cases} \right. \\
& v_{0, \nu}(x)=\left\{\begin{array}{ll}
v_{0}(x), & |x| \leqslant \nu, \\
d_{\nu}, & |x|>\nu,
\end{array} \quad h_{\nu}(t, x)= \begin{cases}h(t, x), & |x| \leqslant \nu, \\
M_{1}(t), & |x|>\nu .\end{cases} \right.
\end{aligned}
$$


Пусть $u_{\nu}=u_{\nu}(t, x), v_{\nu}=v_{\nu}(t, x)$ - о.э.р. задачи $(1),(2)$ с данньпми $u_{0, \nu}, g_{\nu}$ и $v_{0, \nu}, h_{\nu}$ соответственно, а функции $\widetilde{u}_{\nu}(t)$ определены выше. Тогда для любого положительного $h<d_{\nu}-d_{\nu+1}$ для п.в. $t \in(0, T)$

$$
\begin{aligned}
\int_{\mathbb{R}^{n}} & \left(u_{\nu+1}(t, x)-v_{\nu}(t, x)+h\right)^{+} d x \\
= & \int_{\mathbb{R}^{n}}\left(u_{\nu+1}(t, x)-\widetilde{u}_{\nu+1}(t)+\widetilde{u}_{\nu}(t)-v_{\nu}(t, x)+h+d_{\nu+1}-d_{\nu}\right)^{+} d x \\
\leqslant & \int_{\mathbb{R}^{n}}\left|u_{\nu+1}(t, x)-\widetilde{u}_{\nu+1}(t)\right| d x+\int_{\mathbb{R}^{n}}\left|v_{\nu}(t, x)-\widetilde{u}_{\nu}(t)\right| d x \leqslant C_{\nu}+C_{\nu+1}<+\infty
\end{aligned}
$$

с константами $C_{\nu}$, определенными в (16). Как показано выше, из этого неравенства следует (ср. с (18)), что

$$
\mu_{n+1}\left\{(t, x) \in \Pi_{T}: u_{\nu+1}(t, x)>v_{\nu}(t, x)\right\}<\infty
$$

и по предложению 1 В)

$$
\begin{aligned}
\int_{\mathbb{R}^{n}} & \left(u_{\nu+1}(t, x)-v_{\nu}(t, x)\right)^{+} d x \\
\leqslant & \int_{\mathbb{R}^{n}}\left(u_{0, \nu+1}(x)-v_{0, \nu}(x)\right)^{+} d x+\int_{\Pi_{t}}\left(g_{\nu+1}(\tau, x)-h_{\nu}(\tau, x)\right)^{+} d \tau d x \\
= & \int_{|x| \leqslant \nu}\left(u_{0}(x)-v_{0}(x)\right)^{+} d x+\int_{0}^{t} \int_{|x| \leqslant \nu}(g(\tau, x)-h(\tau, x))^{+} d \tau d x .
\end{aligned}
$$

При $\nu \rightarrow \infty$ последовательность $u_{\nu+1}(t, x)-v_{\nu}(t, x)$ сходится к $u(t, x)-v(t, x)$, и, переходя к пределу в (19) с использованием леммы Фату, мы получаем оценку (15). Теорема доказана.

Предположим, что функция $u(t, x) \in L^{\infty}\left(\Pi_{T}\right)$ является наибольшим о.э.р. задачи Коши

$$
u_{t}-\operatorname{div}_{x} \varphi(-u)=-g, \quad u(0, x)=-u_{0}(x) .
$$

Тогда по замечанию 1 b) функция $-u(t, x)$ - наименьшее о.э.р. исходной задачи, и это о.э.р. удовлетворяет свойствам, аналогичным указанным в теореме 1 . Точнее, справедлива следующая

ТЕОрема 1'. Существует наименъиее о.э.p. $u=u(t, x)=J^{-}\left(u_{0}, g\right)$ задачи (1), (2). Кроме того,

1) $u(t, x) \leqslant v(t, x)$ п.в. на $\Pi_{T}$ для любого о.э.супер-р. $v(t, x)$ задачи (1), (2);

2) если $u=J^{-}\left(u_{0}, g\right), v=J^{-}\left(v_{0}, h\right)$, то для п.в. $t \in(0, T)$

$$
\begin{aligned}
\int_{\mathbb{R}^{n}}(u(t, x)-v(t, x))^{+} d x \leqslant & \int_{\mathbb{R}^{n}}\left(u_{0}(x)-v_{0}(x)\right)^{+} d x \\
& +\int_{\Pi_{t}}(g(\tau, x)-h(\tau, x))^{+} d \tau d x .
\end{aligned}
$$


СлЕДСТВИЕ 4. Предположсим, что $u^{ \pm}=J^{ \pm}\left(u_{0}, g\right), v^{ \pm}=J^{ \pm}\left(v_{0}, h\right)$. Тогда

1) для п.в. $t \in(0, T)$

$$
\begin{aligned}
\int_{\mathbb{R}^{n}}\left|u^{ \pm}(t, x)-v^{ \pm}(t, x)\right| d x \leqslant & \int_{\mathbb{R}^{n}}\left|u_{0}(x)-v_{0}(x)\right| d x \\
& +\int_{\Pi_{t}}|g(\tau, x)-h(\tau, x)| d \tau d x
\end{aligned}
$$

(невозрастание расстояния в $L^{1}$ );

2) если $u_{0}(x) \leqslant v_{0}(x)$ n.в. на $\mathbb{R}^{n}, g(t, x) \leqslant h(t, x)$ n.в. на $\Pi_{T}$, то $u^{ \pm}(t, x) \leqslant$ $v^{ \pm}(t, x)$ n.в. на $\Pi_{T}$ (монотонность).

ДокАЗАТЕльство. Для доказательства 1) нужно лишь поменять местами решения $u$ и $v$ в соотношении (15) (и соответственно в (20)) и сложить полученные неравенства. Утверждение 2) непосредственно следует из (15), (20).

Обозначим через $D$ множество пар $\left(u_{0}, g\right)$, где $u_{0} \in L^{\infty}\left(\mathbb{R}^{n}\right), g \in L_{\text {loc }}^{1}\left(\Pi_{T}\right)$ и удовлетворяет условию (3). Пусть $\alpha>0$. Рассмотрим следующий функционал на $D$ :

$$
I_{\alpha}\left(u_{0}, g\right)=\operatorname{essip}_{t \in(0, T)} \int_{\mathbb{R}^{n}}\left(u^{+}(t, x)-u^{-}(t, x)\right) e^{-\alpha|x|} d x, \quad u^{ \pm}=J^{ \pm}\left(u_{0}, g\right) .
$$

Если $u_{0}-v_{0} \in L^{1}\left(\mathbb{R}^{n}\right), g-h \in L^{1}\left(\Pi_{T}\right)$, то, как это следует из $(21), \forall \alpha>0$

$$
\left|I_{\alpha}\left(u_{0}, g\right)-I_{\alpha}\left(v_{0}, h\right)\right| \leqslant 2\left(\left\|u_{0}-v_{0}\right\|_{L^{1}\left(\mathbb{R}^{n}\right)}+\|g-h\|_{L^{1}\left(\Pi_{T}\right)}\right),
$$

т.е. этот функционал непрерывен по Липшицу на $D$ относительно $L^{1}$-метрики. Отсюда легко выводится следующий результат.

ТЕОРема 2. Пусть $D_{u}-$ подмножсество $D$, состоящее из пар $\left(u_{0}, g\right)$, для которых задача (1), (2) однозначно разрешима. Тогда множсество $D_{u}$ замкнуто относительно $L^{1}$-метрики. Другими словами, если последовательность $\left(u_{k}, g_{k}\right) \in D_{u}, k \in \mathbb{N}$, и пара $\left(u_{0}, g\right) \in D$ таковьи, ито $u_{k}-u_{0} \in L^{1}\left(\mathbb{R}^{n}\right)$, $g_{k}-g \in L^{1}\left(\Pi_{T}\right) u u_{k}-u_{0} \rightarrow 0$ в $L^{1}\left(\mathbb{R}^{n}\right), g_{k}-g \rightarrow 0$ в $L^{1}\left(\Pi_{T}\right)$, mo $\left(u_{0}, g\right) \in D_{u}$.

ДокаЗАТЕльство. Пусть $\alpha>0$. Очевидно, $\left(u_{0}, g\right) \in D_{u}$ тогда и только тогда, когда $I_{\alpha}\left(u_{0}, g\right)=0$. Итак, $I_{\alpha}\left(u_{k}, g_{k}\right)=0$ для всех $k \in \mathbb{N}$. Так как $u_{k}-u_{0} \rightarrow 0$ в $L^{1}\left(\mathbb{R}^{n}\right), g_{k}-g \rightarrow 0$ в $L^{1}\left(\Pi_{T}\right)$, из оценки $(22)$ следует, что $I_{\alpha}\left(u_{0}, g\right)=0$.

Как следует из теоремы 2, неединственность о.э.р. задачи (1), (2) сохраняется после достаточно малого возмущения входных данных. Точнее, верно следующее утверждение.

Tеорема 3. Пусть $\left(u_{0}, g\right) \in D \backslash D_{u}, u^{ \pm}=J^{ \pm}\left(u_{0}, g\right)$,

$$
C=\operatorname{essip}_{t \in(0, T)} \int_{\mathbb{R}^{n}}\left(u^{+}(t, x)-u^{-}(t, x)\right) d x \leqslant+\infty .
$$

$E c \Omega u\left(v_{0}, h\right) \in D, u_{0}-v_{0} \in L^{1}\left(\mathbb{R}^{n}\right), \quad g-h \in L^{1}\left(\Pi_{T}\right) \quad u 2\left(\left\|u_{0}-v_{0}\right\|_{L^{1}\left(\mathbb{R}^{n}\right)}+\right.$ $\left.\|g-h\|_{L^{1}\left(\Pi_{T}\right)}\right)<C, m o\left(v_{0}, h\right) \notin D_{u}$. 
ДоказАТЕЛьство. По теореме Леви $C_{\alpha}=I_{\alpha}\left(u_{0}, g\right) \rightarrow C$ при $\alpha \rightarrow 0+$. Поэтому найдется $\alpha>0$ такое, что

$$
2\left(\left\|u_{0}-v_{0}\right\|_{L^{1}\left(\mathbb{R}^{n}\right)}+\|g-h\|_{L^{1}\left(\Pi_{T}\right)}\right)<C_{\alpha}
$$

и по оценке $(22) I_{\alpha}\left(v_{0}, h\right)>0$, т.е. $\left(v_{0}, h\right) \notin D_{u}$.

Заметим, что в примерах неединственности из [7], [9], [10] константа $C=+\infty$. Таким образом, в этих примерах единственность нарушается при любом $L^{1}$-возмушении входных данных.

\section{§3. Единственность о.э.р. в случае периодических входных данных}

Пусть $\xi=\left\{\xi_{1}, \ldots, \xi_{n-1}\right\}$ - система линейно независимых векторов в $\mathbb{R}^{n}$. Функции $u(x) \in L^{\infty}\left(\mathbb{R}^{n}\right), g(t, x) \in L^{\infty}\left(\Pi_{T}\right)$ будем называть $\xi$-периодическими, если $u\left(x+\xi_{i}\right)=u(x)$ п.в. на $\mathbb{R}^{n}$ и соответственно $g\left(t, x+\xi_{i}\right)=g(t, x)$ п.в. на $\Pi_{T}$ для всех $i=1, \ldots, n-1$.

Верна следующая

ЛЕмма 1. Пусть входные данные $u_{0}(x), g(t, x)-\xi$-периодические функиии. Тогда наибольшее и наименьшее о. э.р. задачи (1), (2) также являются $\xi$-периодическими функииями.

ДокАЗАТЕЛЬство. Пусть $u^{+}(t, x)$ - наибольшее о.э.р. задачи (1), (2). Тогда функции $u^{+}\left(t, x \pm \xi_{i}\right), 1 \leqslant i \leqslant n-1$, - о.э.р. этой же задачи ввиду $\xi$-периодичности входных функций $u_{0}(x), g(t, x)$. Так как $u^{+}(t, x)$ - наибольшее о.э.р., верны неравенства $u^{+}\left(t, x+\xi_{i}\right) \leqslant u^{+}(t, x), u^{+}\left(t, x-\xi_{i}\right) \leqslant u^{+}(t, x)$ п.в. на П $T$. Применяя преобразование сдвига к последнему неравенству, запишем его в виде $u^{+}(t, x) \leqslant u^{+}\left(t, x+\xi_{i}\right)$ п.в. на $\Pi_{T}$. Отсюда и из первого неравенства следует, что $u^{+}\left(t, x+\xi_{i}\right)=u^{+}(t, x)$ п.в. на $\Pi_{T}, i=1, \ldots, n-1$. Итак, $u^{+}(t, x)-\xi$-периодическая функция. Аналогично доказьвается $\xi$-периодичность наименьшего о.э.р. $u^{-}(t, x)$.

ТЕОРема 4. О.э.р. задачи (1), (2) с -периодическими входными данными $u_{0}(x), g(t, x)$ единственно.

ДокАЗАТЕльство. Применяя невырожденное линейное преобразование пространственных переменных (см. замечание $1 \mathrm{~d}$ )), сведем задачу к случаю, когда $\xi_{i}=e_{i+1}, i=1, \ldots, n-1,-$ последние $n-1$ канонических базисных векторов пространства $\mathbb{R}^{n}$. Пусть $u^{+}(t, x)$ и $u^{-}(t, x)$ - наибольшее и наименьшее о.э.р. задачи $(1),(2)$. Нужно лишш установить, что эти о.э.р. равны п.в. на $\Pi_{T}$.

Выберем функции $p(y) \in C_{0}^{\infty}\left(\mathbb{R}^{n-1}\right), q(t, s) \in C_{0}^{\infty}\left(\bar{Q}_{T}\right), \bar{Q}_{T}=[0, T) \times \mathbb{R}$, $p, q \geqslant 0, \int_{\mathbb{R}^{n-1}} p(y) d y=1$, и положим $p_{\nu}(y)=\nu^{1-n} p(y / \nu), \nu \in \mathbb{N}$. Так как функции $u^{+}, u^{-}$- о.э.р. задачи (1), (2), они удовлетворяют тождеству (14) из следствия 3 . Применяя разность соответствуюших тождеств к пробной функции $h(t, x)=p_{\nu}\left(x^{\prime}\right) q\left(t, x_{1}\right), x^{\prime}=\left(x_{2}, \ldots, x_{n}\right) \in \mathbb{R}^{n-1}$, получаем следующее 
равенство:

$$
\begin{gathered}
\int_{\Pi_{T}}\left[\left(u^{+}-u^{-}\right) p_{\nu}\left(x^{\prime}\right) q_{t}\left(t, x_{1}\right)+\left(\varphi_{1}\left(u^{+}\right)-\varphi_{1}\left(u^{-}\right)\right) p_{\nu}\left(x^{\prime}\right) q_{x_{1}}\left(t, x_{1}\right)\right] d t d x \\
\quad+\int_{\Pi_{T}} \sum_{i=2}^{n}\left(\varphi_{i}\left(u^{+}\right)-\varphi_{i}\left(u^{-}\right)\right) \nu^{-n} p_{y_{i}}\left(\frac{x^{\prime}}{\nu}\right) q\left(t, x_{1}\right) d t d x=0
\end{gathered}
$$

Запишем (23) в форме

$$
\int_{\mathbb{R}^{n-1}} J_{1}\left(x^{\prime}\right) p_{\nu}\left(x^{\prime}\right) d x^{\prime}+\int_{\mathbb{R}^{n-1}} \sum_{i=2}^{n} J_{i}\left(x^{\prime}\right) \nu^{-n} p_{y_{i}}\left(\frac{x^{\prime}}{\nu}\right) d x^{\prime}=0
$$

где

$$
\begin{aligned}
& J_{1}\left(x^{\prime}\right)=\int_{\bar{Q}_{T}}\left[\left(u^{+}-u^{-}\right) q_{t}\left(t, x_{1}\right)+\left(\varphi_{1}\left(u^{+}\right)-\varphi_{1}\left(u^{-}\right)\right) q_{x_{1}}\left(t, x_{1}\right)\right] d t d x_{1}, \\
& J_{i}\left(x^{\prime}\right)=\int_{\bar{Q}_{T}}\left(\varphi_{i}\left(u^{+}\right)-\varphi_{i}\left(u^{-}\right)\right) q\left(t, x_{1}\right) d t d x_{1}, \quad i=2, \ldots, n
\end{aligned}
$$

и сделаем замену переменных, положив $y=x^{\prime} / \nu$. Получим соотношение

$$
\int_{\mathbb{R}^{n-1}} J_{1}(\nu y) p(y) d y+\frac{1}{\nu} \int_{\mathbb{R}^{n-1}} \sum_{i=2}^{n} J_{i}(\nu y) p_{y_{i}}(y) d y=0
$$

По лемме $1 u^{+}$и $u^{-}$являются $\xi$-периодическими функциями, отсюда следует периодичность функции $J_{1}$. Ясно также, что $J_{i} \in L^{\infty}\left(\mathbb{R}^{n-1}\right), i=1, \ldots, n$. Тогда, как это хорошо известно, последовательность $J_{1}(\nu y)$ сходится $*$-слабо в $L^{\infty}\left(\mathbb{R}^{n-1}\right) \mathrm{k}$ постоянной $C=\int_{P} J_{1}(y) d y$, где $P=[0,1]^{n-1}$, и в пределе при $\nu \rightarrow \infty$ из соотношения (24) следует, что $C=0$, т.е.

$$
\int_{\bar{Q}_{T} \times P}\left[\left(u^{+}-u^{-}\right) q_{t}\left(t, x_{1}\right)+\left(\varphi_{1}\left(u^{+}\right)-\varphi_{1}\left(u^{-}\right)\right) q_{x_{1}}\left(t, x_{1}\right)\right] d t d x=0 .
$$

Выберем константу $M \geqslant \max \left(\left\|u^{+}\right\|_{\infty},\left\|u^{-}\right\|_{\infty}\right)$, и пусть

$$
\omega(r)=\max \left\{\left|\varphi_{1}(u)-\varphi_{1}(v)\right|: u, v \in[-M, M],|u-v| \leqslant r\right\}
$$

- модуль непрерывности функции $\varphi_{1}(u)$ на $[-M, M]$. Функция $\omega(r)$ не убьвает, субаддитивна на $[0,+\infty)$ и $\lim _{r \rightarrow 0+} \omega(r)=\omega(0)=0$. Пусть $\varepsilon>0$. Представим произвольное $r \geqslant 0$ в виде $r=k \varepsilon+s$, где $k \in \mathbb{N} \cup\{0\}$ и $0 \leqslant s<\varepsilon$. Так как $\omega(r)$ не убьвает и субаддитивна, то

$$
\omega(r) \leqslant \omega((k+1) \varepsilon) \leqslant(k+1) \omega(\varepsilon) \leqslant(r+\varepsilon) \frac{\omega(\varepsilon)}{\varepsilon}=C_{\varepsilon}(r+\varepsilon)
$$


при $C_{\varepsilon}=\omega(\varepsilon) / \varepsilon$. Пусть $\chi(s) \in C^{\infty}(\mathbb{R})$ - невозрастающая функция такая, что $\chi(s)=1$ при $s \leqslant R$ и $\chi(s)=0$ при $s \geqslant R+1$ для некоторой положительной константы $R$, а функция $\theta(t) \in C_{0}^{\infty}([0, T))$ такова, что $\theta(t) \geqslant 0, \theta^{\prime}(t) \leqslant 0$. Определим пробную функцию $q\left(t, x_{1}\right)=\chi(s) \theta(t), s=\left|x_{1}\right|-C_{\varepsilon}(T-t)$. Подставив эту пробную функцию в $(25)$, получим соотношение

$$
\begin{aligned}
& \int_{\bar{Q}_{T} \times P}\left(u^{+}-u^{-}\right) \chi(s) \theta^{\prime}(t) d t d x \\
& \quad+\int_{\bar{Q}_{T} \times P}\left[C_{\varepsilon}\left(u^{+}-u^{-}\right)+\left(\varphi_{1}\left(u^{+}\right)-\varphi_{1}\left(u^{-}\right)\right) \operatorname{sign}\left(x_{1}\right)\right] \chi^{\prime}(s) \theta(t) d t d x=0 .
\end{aligned}
$$

Значения $u^{+}(t, x), u^{-}(t, x)$ лежат в $[-M, M]$ для п.в. $(t, x) \in \Pi_{T}$ и по определению $\omega(r)$ и соотношению $(26)$

$$
\left|\varphi_{1}\left(u^{+}\right)-\varphi_{1}\left(u^{-}\right)\right| \leqslant \omega\left(u^{+}-u^{-}\right) \leqslant C_{\varepsilon}\left(u^{+}-u^{-}+\varepsilon\right) \text { п.в. на } \Pi_{T} \text {. }
$$

Поэтому из $(27)$ следует оценка (напомним также, что $\left.\chi^{\prime}(s) \leqslant 0, \theta(t) \geqslant 0, \theta^{\prime}(t) \leqslant 0\right)$

$$
\begin{aligned}
& \int_{[0, T] \times[-R, R] \times P}\left(u^{+}-u^{-}\right)\left|\theta^{\prime}(t)\right| d t d x \\
& \quad \leqslant \int_{\bar{Q}_{T} \times P}\left(u^{+}-u^{-}\right) \chi(s)\left|\theta^{\prime}(t)\right| d t d x \\
& \quad \leqslant \int_{\bar{Q}_{T} \times P}\left[C_{\varepsilon}\left(u^{+}-u^{-}\right)-C_{\varepsilon}\left(u^{+}-u^{-}+\varepsilon\right)\right] \chi^{\prime}(s) \theta(t) d t d x \\
& \leqslant \varepsilon C_{\varepsilon}\|\theta(t)\|_{\infty} \int_{\bar{Q}_{T}}\left|\chi^{\prime}(s)\right| d t d x_{1}=2 T \varepsilon C_{\varepsilon}\|\theta(t)\|_{\infty},
\end{aligned}
$$

поскольку

$$
\begin{aligned}
\int_{\bar{Q}_{T}}\left|\chi^{\prime}(s)\right| d t d x_{1} & =2 \int_{0}^{T} \int_{0}^{+\infty}\left[-\chi^{\prime}\left(x_{1}-C_{\varepsilon}(T-t)\right)\right] d x_{1} d t \\
& =2 \int_{0}^{T} d t=2 T .
\end{aligned}
$$

Так как $\varepsilon C_{\varepsilon}=\omega(\varepsilon) \underset{\varepsilon \rightarrow 0+}{\longrightarrow} 0$, то в пределе при $\varepsilon \rightarrow 0+$ из $(28)$ следует, что

$$
\int_{[0, T] \times[-R, R] \times P}\left(u^{+}-u^{-}\right)\left|\theta^{\prime}(t)\right| d t d x=0
$$

для всех $R>0$ и при любом выборе $\theta$. Отсюда, очевидно, следует требуемое равенство $u^{+}=u^{-}$п.в. на $\Pi_{T}$. Теорема доказана.

СЛЕДСТВИЕ 5 (принцип сравнения). Пусть $v(t, x)$ - о.э.суб-p., $a w(t, x)-$ о.э.супер-р. задачи (1), (2) с входнымми данными $\left(v_{0}(x), g(t, x)\right) u\left(w_{0}(x), h(t, x)\right)$ соответственно, и предположсим, что $v_{0}(x) \leqslant w_{0}(x)$ n.в. на $\mathbb{R}^{n}, g(t, x) \leqslant$ $h(t, x)$ n.в. на $\Pi_{T}$. Eсли по крайней мере одни из данных $\left(v_{0}(x), g(t, x)\right)$ или $\left(w_{0}(x), h(t, x)\right)$ являются $\xi$-периодическими функииями, то $v(t, x) \leqslant w(t, x)$ n.в. на $\Pi_{T}$. 
ДокАЗАТЕЛЬСТво. Пусть $v^{ \pm}(t, x)=J^{ \pm}\left(v_{0}, g\right), w^{ \pm}(t, x)=J^{ \pm}\left(w_{0}, h\right)$. Предположим, что функции $w_{0}(x), h(t, x)-\xi$-периодические. Тогда по следствию 4 и теореме 4

$$
v(t, x) \leqslant v^{+}(t, x) \leqslant w^{+}(t, x)=w^{-}(t, x) \leqslant w(t, x) \text { п.в. на } \Pi_{T} .
$$

Случай $\xi$-периодических функций $v_{0}(x), g(t, x)$ разбирается аналогично:

$$
v(t, x) \leqslant v^{+}(t, x)=v^{-}(t, x) \leqslant w^{-}(t, x) \leqslant w(t, x) \text { п.в. на } \Pi_{T} .
$$

В дополнение к теореме 4 заметим, что в случае $\xi$-периодических данных задачу (1), (2) можно рассматривать как задачу Коши с пространственными переменными на многообразии $T^{n-1} \times \mathbb{R}$, где $T^{n-1}-(n-1)$-мерный тор. Одним из следствий теоремы 4 является существование и единственность о.э.р. этой задачи, понимаемого в смысле [17] (где исследован общий случай произвольного многообразия).

В заключение автор выражает благодарность профессору Филиппу Бенилану за гостеприимство и полезные обсуждения результатов работы во время визита автора в Университет г. Безансон (Франция).

Результаты настоящей статьи предварительно опубликованы в [18].

\section{Список литературы}

1. Кружков C. Н. Обобщенные решения задачи Коши в целом для нелинейных уравнений первого порядка // Докл. АН СССР. 1969. Т. 187. № 1. С. 29-32.

2. Кружков C. Н. Квазилинейные уравнения первого порядка со многими независимыми переменными // Матем. сб. 1970. Т. 81. № 2. С. 228-255.

3. Bénilan Ph. Équation d'évolution dans un space de Banach quelconque et applications // Thèse de Doctorat d'Etat. Centre d'Orsey. Université de Paris-Sud, 1972.

4. Кружков C. Н., Хильдебранд $\Phi$. Задача Коши для квазилинейных уравнений первого порядка в случае, когда область зависимости от начальных данных бесконечна // Вестн. МГУ. Сер. 1. Матем., мех. 1974. №1. С. 93-100.

5. Кружков С. Н., Андреянов П. А. К нелокальной теории задачи Коши для квазилинейных уравнений первого порядка в классе локально-суммируемых функций // Докл. АН CCCP. 1975. T. 220. №1. C. 23-26.

6. Andreianov B.P., Bénilan Ph., Kruzhkov S. N. $L^{1}$-theory of scalar conservation law with continuous flux function // J. Funct. Anal. 2000. V. 171. № 1. P. 15-33.

7. Панов Е. Ю. Обобщенные решения задачи Коши для квазилинейных законов сохранения // Дисс. ... канд. физ.-матем. наук. М.: МГУ, 1991.

8. Панов Е. Ю. О мерозначных решениях задачи Коши для квазилинейного уравнения первого порядка // Изв. РАН. Сер. матем. 1996. Т. 60. № 2. С. 107-148.

9. Кружков C. Н., Панов $E$. Ю. Консервативные квазилинейные законы первого порядка с бесконечной областью зависимости от началњных данных // Докл. АН СССР. 1990. T. 314. № 1. C. $79-84$.

10. Kruzhkov S. N., Panov E. Yu. Osgood's type conditions for uniqueness of entropy solutions to Cauchy problem for quasilinear conservation laws of the first order // Ann. Univ. Ferrara Sez. VII (N.S.). 1996. V. 40 (1994). P. 31-54.

11. Бенилан $\Phi .$, Кружков $C . H$. Квазилинейные уравнения первого порядка с непрерывными нелинейностями // Докл. РАН. 1994. Т. 339. № 2. С. 151-154.

12. Bénilan Ph., Kruzhkov S. N. Conservation laws with continuous flux functions // Nonlinear Differential Equations Appl. 1996. V. 3. P. 395-419. 
13. Barthélemy L. Problème d'obstacle pour une équation quasilinéair du premier ordre // Ann. Fac. Sci. Toulouse Math. (5). 1988. V. 9. № 2. P. 137-159.

14. Barthélemy L., Bénilan Ph. Subsolution for abstract evolution equations // Potential Anal. 1992. V. 1. P. 93-113.

15. Crandall M. G. The semigroup approach to first order quasilinear equations in several space variables // Israel J. Math. 1972. V. 12. P. 108-122.

16. Панов E. Ю. К теории обобщенных энтропийных суб- и супер-решений задачи Коши для квазилинейного уравнения первого порядка // Дифференц. уравнения. 2001. Т. 37. № 2 . C. $249-257$.

17. Панов Е. Ю. О задаче Коши для квазилинейного уравнения первого порядка на многообразии // Диффференц. уравнения. 1997. Т. 33. № 2. С. 257-266.

18. Panov E. Yu. On maximal and minimal generalized entropy solutions to Cauchy problem for a first-order quasilinear equation // Preprint № 2000/26. Laboratoire de mathématiques de Besançon, 2000.

Новгородский государственньй университет

Поступила в редакцию

E-mail: pey@novsu.ac.ru

05.02.2001 